\begin{tabular}{lllllllll} 
O P E R A T I O N S R E S E A R C H A N D D E C I S I O N S \\
\hline No. 2
\end{tabular}

DOI: $10.37190 /$ ord200205

\title{
SATISFACTION OF THE CONDITION OF ORDER PRESERVATION. A SIMULATION STUDY
}

\author{
JIŘí MAZUREK $^{1 *}$, KONRAD KUŁAKOWSKI ${ }^{2}$ \\ ${ }^{1}$ School of Business Administration in Karvina, Silesian University in Opava \\ Univerzitní náměstí 1934/3, 73340 Karviná, Czech Republic \\ ${ }^{2}$ AGH University of Science and Technology, al. Mickiewicza 30, 30-059 Cracow, Poland
}

\begin{abstract}
We examine the satisfaction of the condition of order preservation (COP) concerning different levels of inconsistency for randomly generated multiplicative pairwise comparison matrices (MPCMs) of the order from 3 to 9 , where a priority vector is derived both by the eigenvalue (eigenvector) method (EV) and the geometric mean (GM) method. Our results suggest that the GM method and the EV method preserve the COP almost identically, both for the less inconsistent matrices (with Saaty's consistency index below 0.10), and the more inconsistent matrices (Saaty's consistency index equal to or greater than 0.10). Further, we find that the frequency of the COP violations grows (almost linearly) with the increasing inconsistency of MPCMs measured by Koczkodaj's inconsistency index and Saaty's consistency index, respectively, and we provide graphs to illustrate these relationships.
\end{abstract}

Keywords: pairwise comparisons, eigenvalue method, geometric mean method, condition of order preservation, numerical simulation

\section{Introduction}

Pairwise comparisons can be formally introduced as a binary relation $R$ defined over a finite, non-empty set of objects (concepts). Usually, the relation $R$ has a meaning of "is preferred to", "is more important than", or "is better than", etc. In the multiplicative pairwise comparisons framework, the relation $R$ expresses how many times a concept $i$ is better (more important, more preferred) than a concept $j$. All pairwise comparisons form a $n \times n$ pairwise comparisons (PC) matrix $C=\left[c_{i j}\right]$. A triple $\left(c_{i j}, c_{j k}, c_{i k}\right), i, j, k \in\{1, \ldots, n\}$, is called a triad.

*Corresponding author, email address: mazurek@opf.slu.cz

Received 4 February 2019, accepted 20 July 2020 
Pairwise comparisons belong to the oldest methods of decision making involving a selection of the best object (alternative, criterion, option, etc.) from a finite and nonempty set of objects. Since the number of pairwise comparisons grows as $O\left(n^{2}\right)$, where $n$ denotes the number of objects compared, real-world pairwise comparisons tasks are usually performed for a relatively small number of objects. That is why in our study we focus on pairwise comparisons with $n<10$.

The standard notion of consistency in (multiplicative) pairwise comparisons demands that if an object $\mathrm{A}$ is 4 times better than an object $\mathrm{B}$, which in turn is 2 times better than an object $\mathrm{C}$, then the object $\mathrm{A}$ must be exactly 8 times better than $\mathrm{C}$. This can be considered a "numerical consistency".

In 2008, the condition of order preservation (COP) in the context of pairwise comparisons was introduced by Bana e Costa and Vansnick [2]. The COP states that after comparing a set of objects pairwise, the priority vector (weights associated with every compared object) should not contradict individual judgments. That is if an object $\mathrm{A}$ is directly preferred to an object $\mathrm{B}$, then also the weight of $\mathrm{A}$ should be higher than the weight of $\mathrm{B}$. Further if $\mathrm{A}$ is compared to $\mathrm{B}$, and $\mathrm{C}$ is compared to $\mathrm{D}$, the difference between $\mathrm{A}$ and $\mathrm{B}$ being greater than the difference between $\mathrm{C}$ and $\mathrm{D}$, then also the difference of weights associated with $\mathrm{A}$ and $\mathrm{B}$ should be greater than the difference between $\mathrm{C}$ and $\mathrm{D}$.

This approach to consistency can be labelled as a "preferential consistency" (see Section 3 for more precise definition). After its introduction, the condition of order preservation attracted the attention of several authors, see, e.g., [10, 19, 20, 22].

Studies $[19,20]$ provide a sufficient condition for the COP satisfaction concerning inconsistency expressed by Koczkodaj's inconsistency index both for a multiplicative case and for a more general case based on Alo-groups. The paper [22] proposes a new method for derivation of the priority vector based on the COP. However, little is known about how frequently the COP is satisfied for the eigenvalue (EV) method (also called the eigenvector method) or the geometric mean (GM) method for different inconsistency levels of pairwise comparisons since up to this date, there is no numerical study on the COP published in the literature.

Therefore, the main objective of the paper is to investigate satisfaction/violation of the COP concerning different levels of inconsistency for randomly generated multiplicative pairwise comparison matrices of the order $n=\{3,4, \ldots, 9\}$, where the priority vector is derived both by the EV and by the GM method, to determine which method is better in the sense that which preserves the COP to a larger extent.

Other objectives of the study include the examination of the relationship between the COP violation and the level of inconsistency in general and the relationship between the COP satisfaction and the number of cases guaranteed to be satisfied by two theorems provided in Section 3. Inconsistency of pairwise comparisons is measured via Koczkodaj's inconsistency index $(K I)$ and Saaty's consistency index $(C I)$, respectively. 
The paper is organised as follows: Section 2 provides preliminaries on pairwise comparisons, in Section 3 the condition of order preservation is introduced, Section 4 describes simulation procedure and it is followed by simulations results in Section 5 .

\section{Preliminaries}

The input data for the PC method is a PC matrix $\mathbf{C}=\left[c_{i j}\right]$, where $c_{i j} \in \mathbb{R}_{+}$and $i, j \in\{1, \ldots, n\}$. The values of $c_{i j}$ (and $c_{j i}$ ) indicate the relative importance (or preference) of objects $i$ and $j$.

Definition 1. A matrix $\mathbf{C}=\left[c_{i j}\right]$ is said to be (multiplicatively) reciprocal if

$$
\forall i, j \in\{1, \ldots, n\}: c_{i j}=\frac{1}{c_{j i}}
$$

and $\mathbf{C}=\left[c_{i j}\right]$ is said to be (multiplicatively) consistent if:

$$
\forall i, j, k \in\{1, \ldots, n\}: c_{i j} c_{j k} c_{k i}=1
$$

Since the PC matrix contains subjective judgments of (human) experts, the condition (2) is usually not satisfied. This fact led to the introduction of various inconsistency indices and studies of their properties, see, e.g., [1, 3, 5-8, 15, 18, 21].

Perhaps the best-known inconsistency indices are Saaty's consistency index $C I$ and consistency ratio $C R$, respectively, see [23, 24], and Koczkodaj's inconsistency index $K I$, see [18].

Definition 2. The eigenvalue-based consistency index (Saaty's consistency index) $C I$ of the $n \times n$ reciprocal matrix $\mathbf{C}=\left[c_{i j}\right]$ is equal to

$$
C I(\mathbf{C})=\frac{\lambda_{\max }-n}{n-1}
$$

where $\lambda_{\max }$ is the principal eigenvalue of $\mathbf{C}$.

The value $\lambda_{\max } \geq n$, and $\lambda_{\max }=n$ if and only if $\mathbf{C}$ is consistent [24].

Definition 3. Koczkodaj's inconsistency index $K I$ of $n \times n$ and $(n>2)$ the reciprocal matrix $\mathbf{C}=\left[c_{i j}\right]$ is equal to: 


$$
K I=\max _{i, j, k \in\{1, \ldots, n\}}\left\{\min \left\{\left|1-\frac{c_{i j}}{c_{i k} c_{k j}}\right|,\left|1-\frac{c_{i k} c_{k j}}{c_{i j}}\right|\right\}\right\}
$$

From Definition 3 it follows that $0 \leq K I<1$. The axiomatic characterisation of Koczkodaj's index can be found in [13]. Both Saaty's consistency index and Koczkodaj's index are compared, e.g., in [4].

The result of the pairwise comparisons method is a priority vector (vector of weights) $w$. According to the eigenvalue method proposed by Saaty [23], vector $\mathbf{w}$ is determined as the rescaled principal eigenvector of $\mathbf{C}$. Thus, assuming that $\mathbf{C w}=\lambda_{\max } \mathbf{w}$ the priority vector $\mathbf{w}$ is given as follows:

$$
\mathbf{w}=\gamma\left[w_{1}, \ldots, w_{n}\right]^{T}
$$

where $\gamma$ is a scaling factor. Usually, it is assumed that $\gamma=\left(\sum_{i=1}^{n} w_{i}\right)^{-1}$.

According to the geometric mean method [12], the weight of the $i$ th alternative is given by the geometric mean of the ith row of $\mathbf{C}$. Thus, the priority vector is given as

$$
\mathbf{w}=\gamma\left[\left(\prod_{r=1}^{n} c_{1 r}\right)^{1 / n}, \ldots,\left(\prod_{r=1}^{n} c_{n r}\right)^{1 / n}\right]^{T}
$$

where $\gamma$ is the scaling factor.

In the case of PC matrices of the order $n=3$, the priority vectors $\mathbf{w}$ derived via the GM method and the EV method are identical [11].

\section{Condition of order preservation (COP)}

The condition of order preservation (COP) was introduced in [2].

Definition 4. Let $\mathbf{C}=\left[c_{i j}\right]$ be a pairwise comparison matrix, and let $\mathbf{w}=\left(w_{1}, \ldots, w_{n}\right)$ be a priority vector associated to $\mathbf{C}$. A PC matrix $\mathbf{C}$ is said to satisfy preservation of order preference condition (POP condition) concerning priority vector $\mathbf{w}$ if

$$
c_{i j}>1 \Rightarrow w_{i}>w_{j}
$$

Definition 5. Let $\mathbf{C}=\left[c_{i j}\right]$ be a pairwise comparison matrix, and let $\mathbf{w}=\left(w_{1}, \ldots, w_{n}\right)$ be a priority vector associated to $\mathbf{C}$. A PC matrix $\mathbf{C}$ is said to satisfy preservation of the order of intensity of preference (POIP condition) concerning vector $\mathbf{w}$ if 


$$
c_{i j}>1, c_{k \ell}>1, \text { and } c_{i j}>c_{k \ell} \Rightarrow \frac{w_{i}}{w_{j}}>\frac{w_{k}}{w_{\ell}}
$$

In previous definitions, it is required that relations (6) and (7) be satisfied for all pairs of indices $(i, j)$ and all quadruples of indices $(i, j, k, \ell)$, respectively. In general, there are $\left(n^{2}-n\right)$ individual POP conditions and $\left(n^{2}-n\right)\left(n^{2}-n-2\right)$ individual POIP conditions.

The following two theorems $[19,20]$ provide sufficient conditions to satisfy the POP and POIP conditions, respectively.

Theorem 1. For the PC matrix $\mathbf{C}=\left[c_{i j}\right]$ with Koczkodaj's inconsistency index $K I$ and the ranking vector $\mathbf{w}$ obtained by the EV or GM method it holds that

$$
c_{i j}>\frac{1}{1-K I} \Rightarrow w_{i}>w_{j}
$$

Theorem 2. For the PC matrix $\mathbf{C}=\left[c_{i j}\right]$ with Koczkodaj's inconsistency index $K I$ and the ranking vector $\mathbf{w}$ obtained by the EV or GM method it holds that

$$
\frac{c_{i j}}{c_{k \ell}}>\left(\frac{1}{1-K I}\right)^{2} \Rightarrow \frac{w_{i}}{w_{j}}>\frac{w_{k}}{w_{\ell}}
$$

\section{Numerical simulations}

Numerical (Monte Carlo) simulations belong to popular approaches to the study of pairwise comparisons, see, e.g., [5, 10, 14, 16, 17, 22]. In this study, we perform numerical simulations to examine two main problems:

- how frequently individual POP and POIP conditions are met for different levels of inconsistency concerning the EV and the GM methods,

- how often individual POP and POIP conditions are met for different levels of inconsistency concerning Theorems 1 and 2.

Simulations were performed for pairwise comparisons matrices $\mathbf{C}$ of the order $n\{3,4$, $5,6,7,8,9\}$.

In the beginning, a random pairwise comparisons matrix $n \times n$ was created as follows: 
1. In the first step, the vector $\mathbf{w}=\left(w_{1}, \ldots, w_{n}\right)^{T}$ was randomly drawn, where every $w\left(a_{i}\right) \in[1,9]$.

2. Then, a fully consistent matrix $\mathbf{C}=\left[\frac{w_{i}}{w_{j}}\right]$ was created.

3. Next, for the given disturbance level $1<\gamma<4$ for every entry of $\mathbf{C}$, the actual disturbance coefficient $\delta$ was drawn such that $\delta \in[1 / \gamma, \gamma]$.

4. In the next step, the matrix $\mathbf{C}$ was altered to $\widetilde{\mathbf{C}}=\left[\frac{w_{i}}{w_{j}} \delta_{i j}\right]$ where $\delta_{i j} \in[1 / \gamma, \gamma]$, and for each pair $\{i, j\}$, the value $\delta_{i j}$ was chosen separately.

5. The matrix $\mathbf{C}$ was evaluated for the POP and POIP conditions and inconsistency ( $K I$ and $C I$ indices) by the EV or GM method.

For every matrix size $3 \times 3$ to $9 \times 9$ and every disturbance level ( 300 levels), 100 or 500 random matrices were generated (210 000 or 1050000 matrices, respectively, in total). The results have a form of an Excel file and can be freely downloaded from the Mendeley data storage [25].

\section{Results}

Table 1 provides the average percentage of satisfied individual POP and POIP conditions for multiplicative pairwise comparison matrices of the order $3 \leq n \leq 9$, when the priority vector (vector of weights) was elicited via EV method and GM method, whilst $C I<0.10$ (matrices that can be considered "less inconsistent"). It can be seen that individual POP conditions were satisfied in approximately $90 \%$ of all cases, and individual POIP conditions in approximately $96 \%$ of all cases.

Table 1. Satisfaction of POP and POIP conditions [\%], $C I<0.10$.

EV method was used for the last two columns

\begin{tabular}{|c|c|c|c|c|c|c|}
\hline$n$ & POP (EV) & POP (GM) & POIP (EV) & POIP (GM) & POP Th1 & POIP Th2 \\
\hline 3 & 91.02 & 91.02 & 96.60 & 96.60 & 58.25 & 7.60 \\
\hline 4 & 90.44 & 90.59 & 95.79 & 95.79 & 40.39 & 3.50 \\
\hline 5 & 89.70 & 89.88 & 95.80 & 95.82 & 32.90 & 2.53 \\
\hline 6 & 89.91 & 90.01 & 95.80 & 95.82 & 29.41 & 2.25 \\
\hline 7 & 89.66 & 89.74 & 95.83 & 95.85 & 27.10 & 2.12 \\
\hline 8 & 89.57 & 89.64 & 95.98 & 96.00 & 26.45 & NA \\
\hline 9 & 89.62 & 89.70 & 96.03 & 96.05 & 25.28 & NA \\
\hline
\end{tabular}


Table 2 provides analogous data for matrices with $C I \geq 0.10$ (matrices that can be considered "more inconsistent"). It is clear that more inconsistent matrices are worse in satisfaction of the POP and POIP conditions by several percent.

Table 2. Satisfaction of POP and POIP conditions (in \%), $C I \geq 0.10$.

EV method was used for the last two columns

\begin{tabular}{|c|c|c|c|c|c|c|}
\hline$n$ & POP (EV) & POP (GM) & POIP (EV) & POIP (GM) & POP Th1 & POIP Th2 \\
\hline 3 & 87.33 & 87.33 & 96.00 & 96.00 & 45.83 & 5.29 \\
\hline 4 & 85.88 & 86.29 & 94.01 & 94.03 & 21.30 & 0.53 \\
\hline 5 & 83.64 & 84.21 & 93.63 & 93.69 & 9.72 & 0.05 \\
\hline 6 & 82.70 & 83.06 & 93.56 & 93.64 & 5.45 & 0.01 \\
\hline 7 & 82.01 & 82.55 & 93.43 & 93.50 & 3.27 & 0.00 \\
\hline 8 & 81.71 & 82.18 & 93.48 & 93.55 & 2.22 & NA \\
\hline 9 & 81.37 & 81.83 & 93.40 & 93.46 & 1.52 & NA \\
\hline
\end{tabular}

The last two columns in Tables 1 and 2 provide the percentage of consistent matrices satisfying Theorems 1 and 2. As can be seen, both Theorems "capture" more cases for matrices with $C I<0.10$ and $n=\{3,4\}$. As for the comparison between EV method and GM method, it is evident that both methods yield almost identical results (the GM method is better by approximately $0.1 \%$ ).

The relationship between average number of violated POP and POIP individual conditions with respect to Koczkodaj's index $K I$ and $n=\{4,7,9\}$ and EV method is shown in Figs. 1-6 (each point represents one of 300 disturbance levels, see previous section, step 3). It can be seen that the number of individual violations of the POP and POIP conditions grows with increasing $K I$ roughly linearly. Figures $7-10$ show average numbers of matrix entries satisfying POP and POIP individual conditions via Theorems 1 and 2 with

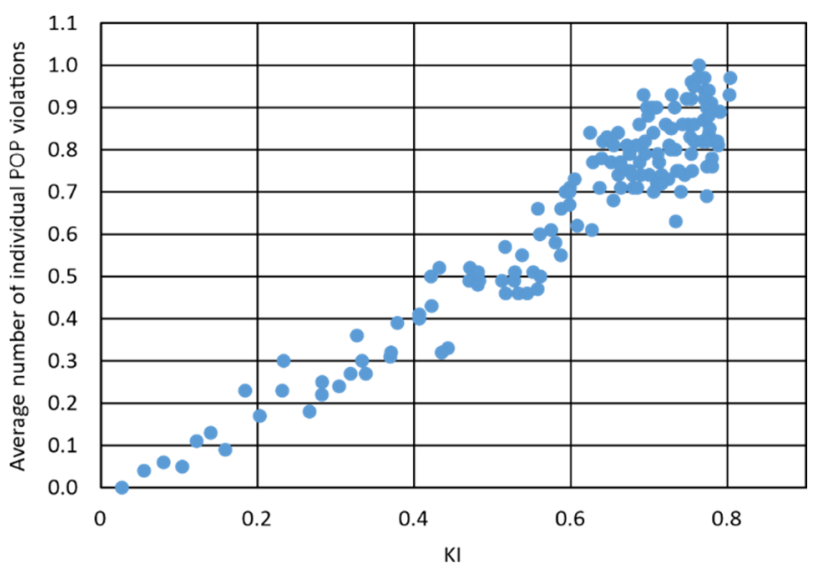

Fig. 1. Average number of individual POP violations (EVM), $n=4$ 


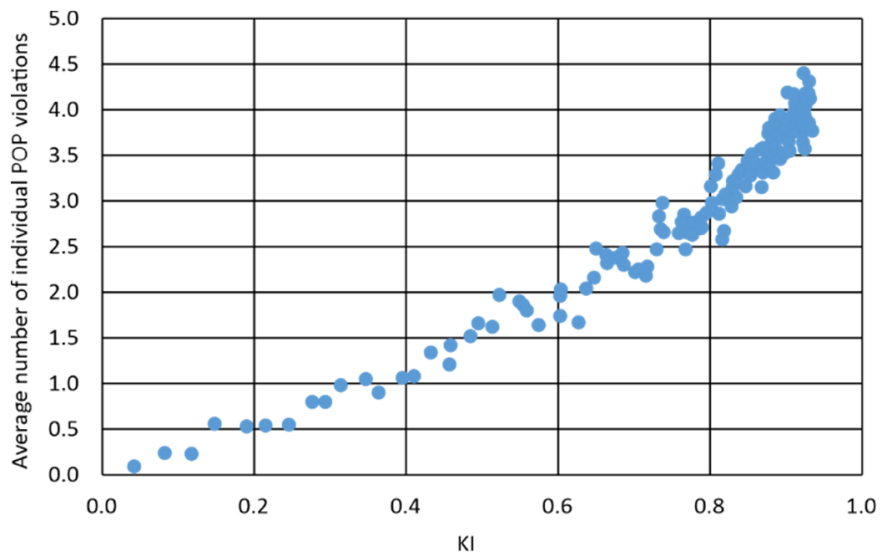

Fig. 2. Average number of individual POP violations (EVM), $n=7$

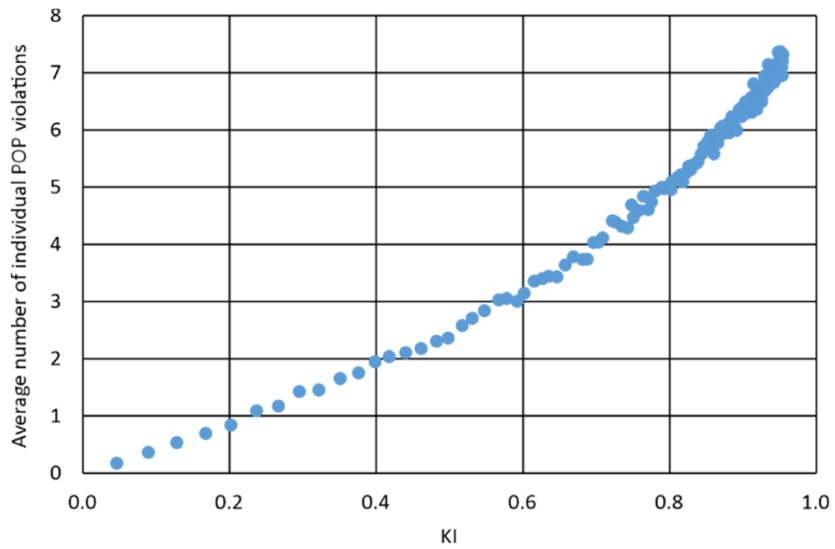

Fig. 3. Average number of individual POP violations (EVM), $n=9$

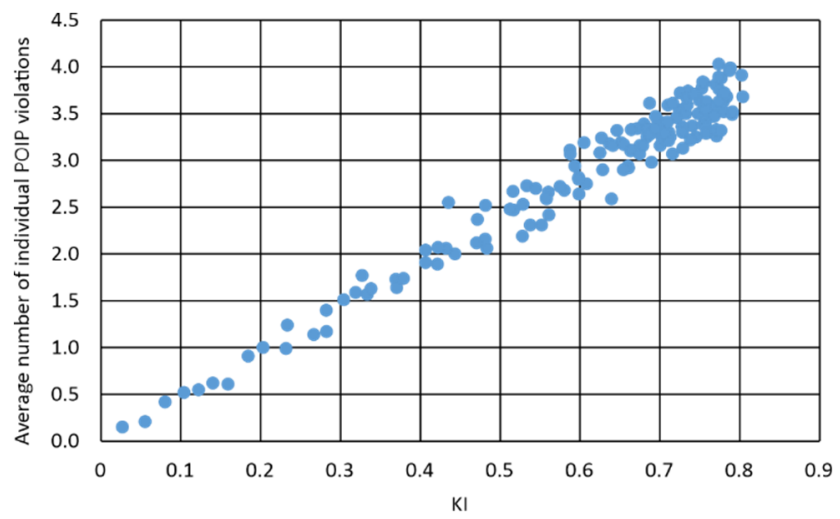

Fig. 4. Average number of individual POIP violations (EVM), $n=4$ 


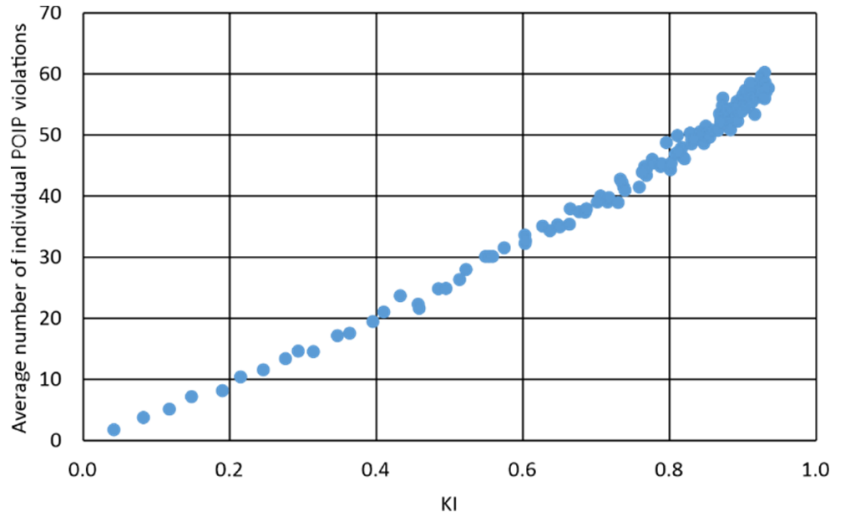

Fig. 5. Average number of individual POIP violations (EVM), $n=7$

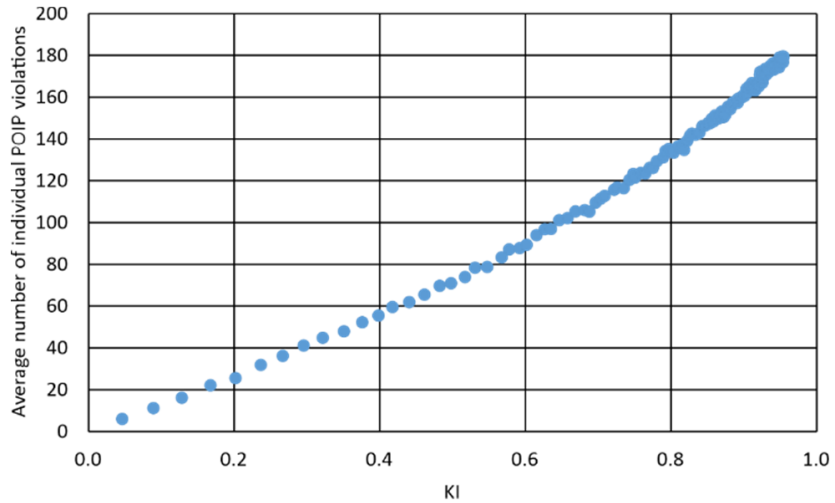

Fig. 6. Average number of individual POIP violations (EVM), $n=9$

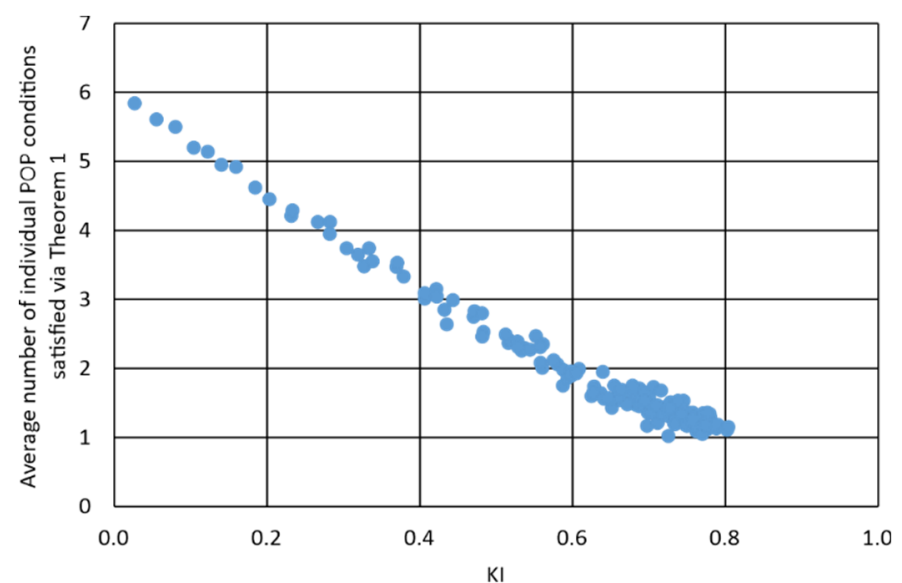

Fig. 7. Average number of inidvidual POP satisfied via Theorem $1(\mathrm{EVM}), n=4$ 


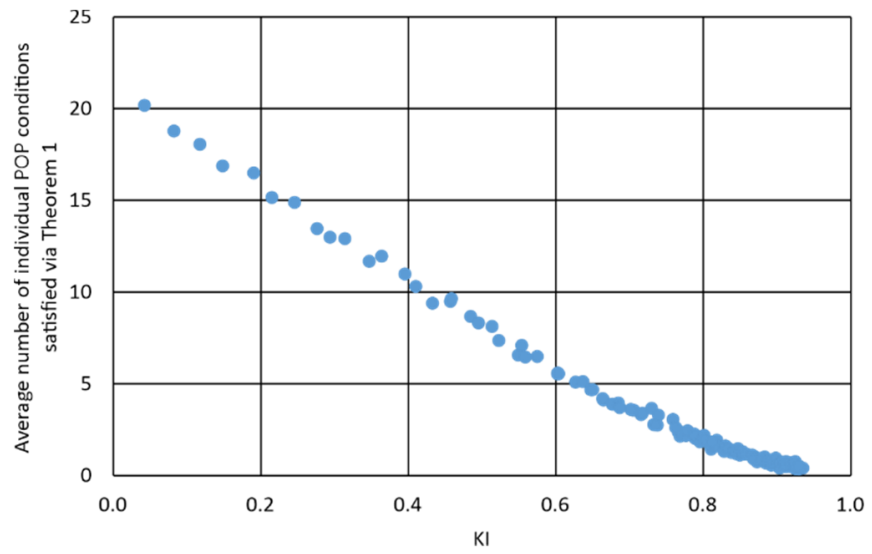

Fig. 8. Average number of individual POP satisfied via Theorem 1 (EVM), $n=7$

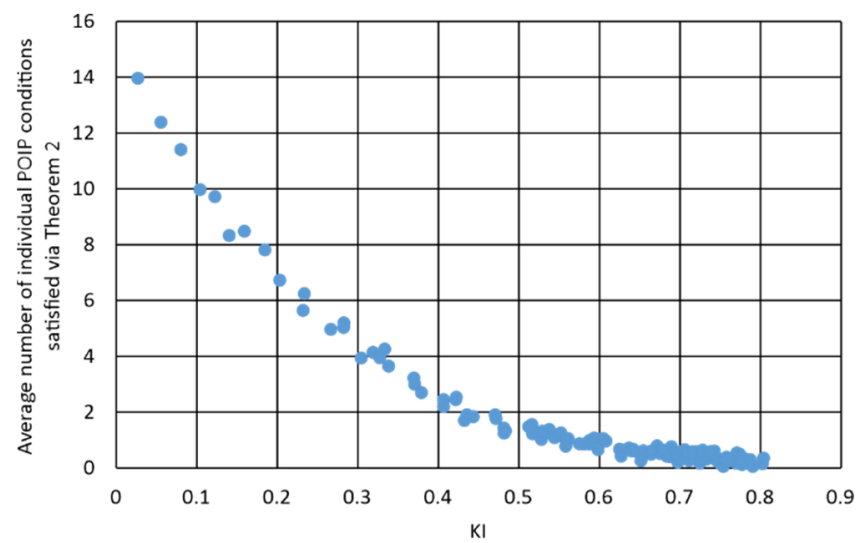

Fig. 9. Average number of individual POIP satisfied via Theorem 2 (EVM), $n=4$

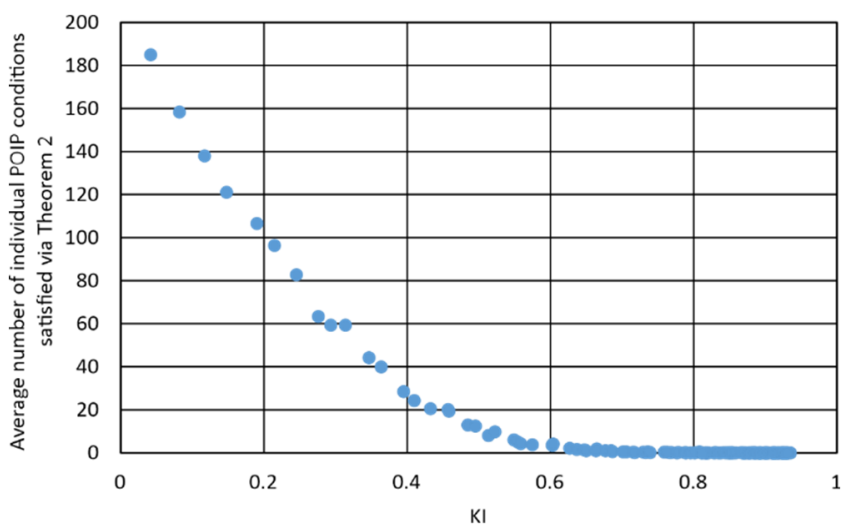

Fig. 10. Average number of individual POIP satisfied via Theorem 2 (EVM), $n=7$ 
respect to $K I$ and $E V$ method for $n=\{4,7\}$. The number of cases captured by Theorems 1 and 2 decreases approximately linearly (for POP) and hyperbolically (for POIP) with the growing $K I$.

Since the GM method satisfies the COP (for a given matrix order) in a higher percentage than the EV method (see Tables 1 and 2), a question arises whether the EV method always satisfies the COP if the GM method satisfies the COP. The next proposition provides the answer to this question with respect to the POP condition.

Proposition 1. Let $E$ denote the set of all PCMs of a given order $n \in N, n>2$ satisfying the POP condition where the priority vector is determined via the EV method. Let $G$ denote the set of all PCMs of a given order $n$ satisfying the POP condition where the priority vector is determined by the GM method. Then, there exists at least one $n$ such that $E \not \subset G$.

Proof. We find a PC matrix $\mathbf{A}$ such that $\mathbf{A} \in E$, but $\mathbf{A} \notin G$, hence $E \not \subset G$. Consider the following PC matrix $\mathbf{A}$ :

$$
A=\left[\begin{array}{cccc}
1 & \frac{1}{6} & 3 & 3 \\
6 & 1 & 6 & 9 \\
\frac{1}{3} & \frac{1}{6} & 1 & 1 \\
\frac{1}{3} & \frac{1}{9} & 1 & 1
\end{array}\right]
$$

The priority vector $\mathbf{w}_{\mathrm{GMM}}(\mathbf{A})=(0.161,0.393,0.393,0.053)$. When checking individual POP conditions, we see that $a_{23}=3$, hence $w_{2}$ should be greater than $w_{3}$, however $w_{2}=w_{3}$. Therefore, the POP condition for $\mathbf{A}$ with respect to the GM method is violated. The priority vector $\mathbf{w}_{\mathrm{EVM}}(\mathbf{A})=(0.159,0.412,0.386,0.043)$. We check the condition for $a_{23}=3$ again: $w_{2}>w_{3}$ is satisfied, and it is easy to check that all other individual POP conditions are satisfied as well. Hence, the POP condition for A with respect to the EV method does hold. Naturally, it is possible that $E \subseteq G$ for some $n \in N, n \neq 4$. Also, whether similar statement to Proposition 1 holds for the POIP condition or the COP (the POP and POIP condition together) remains unknown and certainly worth investigating in the future.

\section{Conclusions}

The aim of the paper was to investigate how frequently the condition of order preservation (COP) is satisfied or violated for randomly generated multiplicative 
pairwise comparison matrices up to the order of $n=9$ concerning their inconsistency expressed by Koczkodaj's inconsistency index and Saaty's consistency index for both EV and GM method.

Numerical results suggest that the number of violations of individual POP and POIP conditions grows with the growing inconsistency, and the geometric mean method and the eigenvalue method yield almost identical results, therefore, for practical purposes, the priorisation method in the COP framework does not matter. Also, it was found that the average number of individual POP conditions guaranteed by Theorem 1 decreases roughly linearly, and the average number of individual POIP conditions guaranteed by Theorem 2 decreases roughly hyperbolically. Both theorems were significantly more efficient (captured significantly more cases) for smaller matrices (of the order $n=\{3,4\}$ ) and less inconsistent matrices (with $C I<0.10$ ), than for larger $(n \geq 5)$ and more inconsistent matrices (with $C I \geq 0.10)$ ).

Further research may examine differences between EV and GM methods, other aspects of the COP, such as the maximum of possible individual violations, or the relationship between "numerical consistency" and "preferential consistency" in general.

\section{Acknowledgment}

The research was partially supported by the project GACR No. 18-01246S.

\section{References}

[1] Aguaron J., Moreno-Jimenez J.M., The geometric consistency index: Approximated threshold, Eur. J. Oper. Res., 2003, 147 (1), 137-145.

[2] BANA E COSTA C.A., VANSNICK J., A critical analysis of the eigenvalue method used to derive priorities in AHP, Eur. J. Oper. Res., 2008, 187 (3), 1422-1428.

[3] BARZILAI J., Consistency measures for pairwise comparison matrices, J. Multi-Crit. Dec. Anal., 7 (3), 1998, 123-132.

[4] BozÓKI S., RAPCSÁK T., On Saaty's and Koczkodaj's inconsistencies of pairwise comparison matrices, J. Global Opt., 2008, 42 (2), 157-175.

[5] Brunelli M., Canal L., Fedrizzi M., Inconsistency indices for pairwise comparison matrices: a numerical study, Ann. Oper. Res., 2013, 211 (1), 493-509.

[6] BRUNELli M., FEDRIZZI M., Axiomatic properties of inconsistency indices for pairwise comparisons, J. Oper. Res. Soc., 2015, 66 (1), 1-15.

[7] BRUNELli M., Studying a set of properties of inconsistency indices for pairwise comparisons, Ann. Oper. Res., 2017, 248 (1-2), 143-161.

[8] BRUNELli M., A survey of inconsistency indices for pairwise comparisons, Int. J. Gen. Syst., 2018, 47 (8), 751-771.

[9] CaVallo B., Functional relations and Spearman correlation between consistency indices, J. Oper. Res. Soc., 2020, 71 (2), 301-311.

[10] Cavallo B., D’apuzzo L., Preservation of preferences intensity of an inconsistent pairwise comparison matrix, Int. J. Appr. Reas., 2020, 116, 33-42. 
[11] Crawford G., Williams C., A note on the analysis of subjective judgment matrices, J. Math. Psych., 1985, 29 (4), 387-405.

[12] CRAWFORD G.B., The geometric mean procedure for estimating the scale of a judgement matrix, Math. Model., 1987, 9 (3), 327-334.

[13] Csató L., Characterization of an inconsistency ranking for pairwise comparison matrices, Ann. Oper. Res., 2018, 261 (1-2), 155-165.

[14] Csató L., Petróczy D.G., Rank monotonicity and the eigenvector method, Manuscript, 2020, ArXiv: 1902.10790.

[15] Golden B., Wang Q., An alternate measure of consistency, [In:] B. Golden, E. Wasil, P.T. Harker (Eds.), The Analytic Hierarchy Process, Applications and Studies, Springer-Verlag, Berlin 1989, 68-81.

[16] Herman M.W., Koczkodau W.W., A Monte Carlo Study of Parwise Comparison, Inf. Proc. Lett., 1996, 57, 25-29.

[17] IshiZAKa A., Lusti M., How to derive priorities in AHP: a comparative study, Central Eur. J. Oper. Res., 2006, 14 (4), 387-400.

[18] Koczkodau W.W., A new definition of consistency of pairwise comparisons, Math. Comp. Model., 1993, 18 (7), 79-84.

[19] KulakOWSKI K., Notes on Order Preservation and Consistency in AHP, Eur. J. Oper. Res., 2015, 245, 333-337.

[20] Kulakowski K., MazureK J., Ramík J., Soltys M., When is the condition of preservation met?, Eur. J. Oper. Res., 2019, 277, 248-254.

[21] MAZUReK J., Some notes on the properties of inconsistency indices in pairwise comparisons, Oper. Res. Dec., 2018, 1, 27-42.

[22] MazureK J., RAmíK J., Some new properties of inconsistent pairwise comparison matrices, Int. J. Appr. Reas., 2019, 113, 119-132.

[23] SAATY T.L., A scaling method for priorities in hierarchical structures, J. Math. Psych., 1977, 15 (3), 234-281.

[24] SaAty T.L., Analytic Hierarchy Process, McGraw-Hill, New York 1980.

[25] https://data.mendeley.com/datasets/kskpwfcf9z/1 